\title{
TWO-DIMENSIONAL GOLOD COMPLEXES
}

\author{
KOUYEMON IRIYE AND DAISUKE KISHIMOTO
}

\begin{abstract}
We characterize two-dimensional Golod complexes combinatorially by vertexbreakability and topologically by the fat-wedge filtration of a polyhedral product. Applying the characterization, we consider a difference between Golodness over fields and rings, which enables us to give a two-dimensional simple Golod complex over any field such that the corresponding moment-angle complex is not a suspension.
\end{abstract}

\section{INTRODUCTION}

Throughout this paper, let $K$ denote a simplicial complex over the vertex set $[m]=$ $\{1,2, \ldots, m\}$, where ghost vertices are not allowed. Recall that the Stanley-Reisner ring of $K$ over a commutative ring $R$ is defined by

$$
R[K]=R\left[v_{1}, \ldots, v_{m}\right] /\left(v_{i_{1}} \cdots v_{i_{k}} \mid\left\{i_{1}, \ldots, i_{k}\right\} \notin K\right)
$$

where we assume that each $v_{i}$ is of degree 2. It is of particular interest to give a characterization of $K$ which is equivalent to a given algebraic property of $R[K]$. For instance, Cohen-Macaulayness of $R[K]$ is completely characterized by a homological property of $K$. In this paper, we consider a property of $R[K]$, called Golodness, where Golodness was first introduced for a noetherian local ring [3].

Definition 1.1. A simplicial complex $K$ is called Golod over $R$ if all products and (higher) Massey products in $\operatorname{Tor}_{+}^{R\left[v_{1}, \ldots, v_{m}\right]}(R[K], R)$ vanish, where products and (higher) Massey products are given by the Koszul complex of $R[K]$.

We simply say that $K$ is Golod if it is Golod over any ring.

Baskakov, Buchstaber and Panov [2] showed that there is a space $Z_{K}$, called the moment-angle complex for $K$, such that

$$
H^{*}\left(Z_{K} ; R\right) \cong \operatorname{Tor}_{*}^{R\left[v_{1}, \ldots, v_{m}\right]}(R[K], R)
$$

where the isomorphism respects products and (higher) Massey products. This adds a topological viewpoint to the study of Stanley-Reisner rings, which is particularly useful in studying Golodness because $K$ is Golod if $Z_{K}$ is a suspension. The authors [9] developed a nice technology for the study of the homotopy type of $Z_{K}$, or more generally

2010 Mathematics Subject Classification. 13F55, 55P15.

Key words and phrases. Stanley-Reisner ring, Golod property, neighborly complex, polyhedral product, fat-wedge filtration. 
a polyhedral product, which is called the fat-wedge filtration. In particular, the following is proved in [9], where $\mathbb{R} Z_{K}$ denotes the real moment-angle complex for $K$.

Theorem 1.2. If the fat-wedge filtration of $\mathbb{R} Z_{K}$ is trivial, then $Z_{K}$ is a suspension, implying $K$ is Golod.

For several important Golod complexes such as the Alexander dual of sequentially Cohen-Macaulay complexes, the fat-wedge filtration of $\mathbb{R} Z_{K}$ has been proved to be trivial $[7,8,9]$. In particular, by describing a condition for the fat-wedge filtration of $\mathbb{R} Z_{K}$ combinatorially, combinatorial characterizations for Golodness of 1-dimensional complexes and triangulations of closed surfaces have been obtained in [7, 9]. Here we recall the result on surface triangulations. We say that $K$ is $k$-neighborly if every $k+1$ vertices of $K$ form a simplex of $K$.

Theorem 1.3. If $K$ is a triangulation of a connected closed surface, then the following conditions are equivalent:

(1) $K$ is Golod;

(2) $K$ is 1-neighborly;

(3) the fat-wedge filtration of $\mathbb{R} Z_{K}$ is trivial.

In this paper, we extend this result to all 2-dimensional simplicial complexes. Clearly, general 2-dimensional simplicial complexes are much more complicated than surface triangulations, and so neighborliness may not be enough to characterize Golodness of 2-dimensional simplicial complexes. Indeed, we have the following example.

Example 1.4. Let $K$ be a wedge of two copies of the boundary of a 3-simplex. By definition, $K$ is not 1-neighborly. On the other hand, it follows from [9, Corollary 7.5] that $Z_{K}$ is of the homotopy type of a wedge of spheres. Then $K$ is Golod.

Thus we need to consider a new notion to characterize Golodness of 2-dimensional simplicial complexes. Recall that the full subcomplex of $K$ over a non-empty subset $I \subset[m]$ is defined by $K_{I}=\{\sigma \in K \mid \sigma \subset I\}$. For a vertex $v \in K$, we write $K_{[m]-v}$ by $\mathrm{dl}_{K}(v)$. Now we introduce a new notion.

Definition 1.5. We say that $K$ has vertex-breakable $n$-th homology over an abelian group $A$ if the map

$$
\bigoplus_{v \in[m]}\left(i_{v}\right)_{*}: \bigoplus_{v \in[m]} H_{n}\left(\mathrm{dl}_{K}(v) ; A\right) \rightarrow H_{n}(K ; A)
$$

is not surjective, where $i_{v}: \mathrm{dl}_{K}(v) \rightarrow K$ denotes the inclusion. We simply say that $K$ has vertex-breakable $n$-th homology if $K$ has vertex-breakable $n$-th homology over some finitely generated abelian group.

Example 1.6. If $K$ is a triangulation of a connected closed $n$-manifold, then $K$ has vertex-breakable $n$-th homology. 
Recall that a graph is called chordal if its minimal cycles are of length three. Now we state our results.

Theorem 1.7. If $K$ is a two-dimensional simplicial complex, then the following conditions are equivalent:

(1) $K$ is Golod;

(2) the 1-skeleton of $K$ is chordal, and every full subcomplex of $K$ having vertexbreakable second homology is 1-neighborly;

(3) the fat-wedge filtration of $\mathbb{R} Z_{K}$ is trivial.

Golodness was originally defined for local rings by a certain equality involving the Poincaré series of their cohomology. Later, Golod [3] proved that the equality is equivalent to vanishing of products and (higher) Massey products as in Definition 1.1, which enables us to generalize the notion of Golodness over rings. Then it is natural to ask whether or not Golodness over fields and rings are different. Applying Theorem 1.7, we will prove that there is certainly a difference between them.

Theorem 1.8. There is a two-dimensional simplicial complex which is Golod over any field but is not Golod over some ring.

As in Theorems 1.3 and 1.7 as well as [4, 5, 7, 8, 9], Golodness over any field of several important classes of simplicial complexes has been proved to be a consequence of the corresponding moment-angle complexes being suspensions. Then it is natural to ask whether or not there is a simplicial complex $K$ such that $K$ is Golod over any field and $Z_{K}$ is not a suspension. Yano and the first author [10] proved that there is such a simplicial complex by a direct calculation. We show that a simplicial complex of Theorem 1.8 is such a simplicial complex too, which is drastically simpler than the one of Yano and the first author.

Corollary 1.9. There is a two-dimensional simplicial complex $K$ such that $K$ is Golod over any field and $Z_{K}$ is not a suspension.

Proof. Let $K$ be a simplicial complex of Theorem 1.8. The first statement follows from Theorem 1.8. If $Z_{K}$ is a suspension, then by Theorem 1.2, $K$ must be Golod over any ring. Thus the second statement also follows from Theorem 1.8.

Section 2 recalls properties of the fat-wedge filtration of a polyhedral product that we are going to use, and Section 3 considers a relation between Golodness and vertexbreakability. Section 4 proves Theorem 1.7, and Section 5 constructs a triangulation $M$ of the Moore space $S^{1} \cup_{4} e^{2}$ which proves Theorem 1.8.

Acknoledgement: The authors were supported by JSPS KAKENHI No. $19 K 03473$ and No. 17K05248. 


\section{FAt-WEdGe FiLtration}

In this section, we recall from [9] properties of the fat-wedge filtration of a polyhedral product that we are going to use. First, we define a polyhedral product. Let $(\underline{X}, \underline{A})=$ $\left\{\left(X_{i}, A_{i}\right)\right\}_{i=1}^{m}$ be a collection of pairs of spaces. For a subset $\sigma \subset[m]$, let

$$
(\underline{X}, \underline{A})^{\sigma}=Y_{1} \times \cdots \times Y_{m}, \quad \text { where } \quad Y_{i}= \begin{cases}X_{i} & i \in \sigma \\ A_{i} & i \notin \sigma .\end{cases}
$$

The polyhedral product of $(\underline{X}, \underline{A})$ over $K$ is defined by

$$
Z_{K}(\underline{X}, \underline{A})=\bigcup_{\sigma \in K}(\underline{X}, \underline{A})^{\sigma} .
$$

Clearly, $Z_{K}(\underline{X}, \underline{A})$ is natural with respect to $(\underline{X}, \underline{A})$ and inclusions of subcomplexes of $K$. In particular, for $\emptyset \neq I \subset[m], Z_{K_{I}}\left(\underline{X}_{I}, \underline{A}_{I}\right)$ is assumed to be a subspace of $Z_{K}(\underline{X}, \underline{A})$, where $\left(\underline{X}_{I}, \underline{A}_{I}\right)=\left\{\left(X_{i}, A_{i}\right)\right\}_{i \in I}$. For a collection of pointed spaces $\underline{X}=\left\{X_{i}\right\}_{i=1}^{m}$, let $(C \underline{X}, \underline{X})=\left\{\left(C X_{i}, X_{i}\right)\right\}_{i=1}^{m}$. The polyhedral product $Z_{K}(C \underline{X}, \underline{X})$ is particularly important. Indeed, the moment-angle complex and the real moment-angle complex for $K$ are defined by

$$
Z_{K}=Z_{K}\left(D^{2}, S^{1}\right) \text { and } \mathbb{R} Z_{K}=Z_{K}\left(D^{1}, S^{0}\right),
$$

which play the fundamental role in toric topology. We refer to a comprehensive survey [1] for basic properties of polyhedral products.

Next we define the fat-wedge filtration of $Z_{K}(\underline{X}, \underline{A})$. Let

$$
Z_{K}^{i}(\underline{X}, \underline{A})=\left\{\left(x_{1}, \ldots, x_{m}\right) \in Z_{K}(\underline{X}, \underline{A}) \mid \text { at least } m-i \text { of } x_{k} \text { are basepoints }\right\}
$$

for $0 \leq i \leq m$. Clearly,

$$
Z_{K}^{i}(\underline{X}, \underline{A})=\bigcup_{I \subset[m],|I|=i} Z_{K_{I}}\left(\underline{X}_{I}, \underline{A}_{I}\right) .
$$

The following is proved in [9, Theorem 3.1].

Theorem 2.1. For each $\emptyset \neq I \subset[m]$, there is a map $\varphi_{K_{I}}:|K| \rightarrow \mathbb{R} Z_{K_{I}}^{|I|-1}$ such that

$$
\mathbb{R} Z_{K}^{i}=\mathbb{R} Z_{K}^{i-1} \bigcup_{I \subset[m],|I|=i} C\left|K_{I}\right|
$$

where the attaching maps are $\varphi_{K_{I}}$.

By the construction [9, Section 5] of $\varphi_{K}$, we have the following naturality.

Lemma 2.2. Let $L$ be a subcomplex of $K$ such that the vertex set of $L$ is the same as $K$. Then there is a commutative diagram

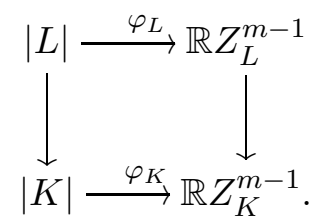


We say that the fat-wedge filtration of $\mathbb{R} Z_{K}$ is trivial if $\varphi_{K_{I}}$ is null-homotopic for each $\emptyset \neq I \subset[m]$. The main property of the fat-wedge filtration that we use is the following [9, Theorem 1.2], and Theorem 1.2 is its immediate corollary.

Theorem 2.3. If the fat-wedge filtration of $\mathbb{R} Z_{K}$ is trivial, then for any $\underline{X}=\left\{X_{i}\right\}_{i=1}^{m}$, there is a homotopy equivalence

$$
Z_{K}(C \underline{X}, \underline{X}) \simeq \bigvee_{\emptyset \neq I \subset[m]}\left|\Sigma K_{I}\right| \wedge \widehat{X}^{I}
$$

where $\widehat{X}^{I}=\bigwedge_{i \in I} X_{i}$.

To show that the map $\varphi_{K}$ is null-homotopic, the following property is useful, which is proved in the proof of [9, Theorem 7.2]. Recall that a subset $\sigma \subset[m]$ is called a minimal non-face of $K$ if $\sigma \notin K$ and $\sigma-i \in K$ for all $i \in \sigma$.

Lemma 2.4. Let $\widehat{K}$ be a simplicial complex obtained by adding all minimal non-faces to $K$. Then the map $\varphi_{K}$ factors through the inclusion $|K| \rightarrow|\widehat{K}|$.

We will use the following criterion for triviality of the fat-wedge filtration of $\mathbb{R} Z_{K}$ [9, Theorem 1.6].

Theorem 2.5. If $K$ is $\left\lceil\frac{\operatorname{dim} K}{2}\right\rceil$-neighborly, then the fat-wedge filtration of $\mathbb{R} Z_{K}$ is trivial.

\section{VerteX-BREAKABILITY}

In this section, we prove a relation between vertex-breakability and Golodness. To this end, we recall a combinatorial description of the product in $\operatorname{Tor}_{*}^{R\left[v_{1}, \ldots, v_{m}\right]}(R[K], R)$. By the classical theorem of Hochster, there is an isomorphism of $R$-modules

$$
\operatorname{Tor}_{i}^{R\left[v_{1}, \ldots, v_{m}\right]}(R[K], R) \cong \bigoplus_{\emptyset \neq I \subset[m]} \widetilde{H}^{i-|I|-1}\left(K_{I} ; R\right) .
$$

It is remarkable that Baskakov, Buchstaber and Panov [2] proved that the product in $\operatorname{Tor}_{*}^{R\left[v_{1}, \ldots, v_{m}\right]}(R[K], R)$ is nicely described through this isomorphism as follows. For disjoint simplicial complexes $K, L$, let $K * L$ denote the join of $K$ and $L$, that is,

$$
K * L=\{\sigma \sqcup \tau \mid \sigma \in K, \tau \in L\} .
$$

Then $|K * L|=|K| *|L| \simeq \Sigma|K| \wedge|L|$. For $\emptyset \neq I, J \subset[m]$, we define a map $m_{I, J}: K_{I \cup J} \rightarrow$ $K_{I} * K_{J}$ by

$$
m_{I, J}(\sigma)=\sigma_{I} \sqcup \sigma_{J}
$$

for $I \cap J=\emptyset$ and $m_{I, J}=*$ for $I \cap J \neq \emptyset$.

Theorem 3.1. The product in $\operatorname{Tor}_{*}^{R\left[v_{1}, \ldots, v_{m}\right]}(R[K], R)$ is identified with

$$
m_{I, J}^{*}: \widetilde{H}^{i-|I|-1}\left(K_{I} ; R\right) \otimes \widetilde{H}^{j-|J|-1}\left(K_{J} ; R\right) \rightarrow \widetilde{H}^{i+j-|I|-|J|-1}\left(K_{I \cup J} ; R\right) .
$$

We consider a map which is trivial in cohomology. 
Lemma 3.2. Let $f: X \rightarrow Y$ be a map between $C W$-complexes of finite type. If a map $f^{*}: H^{n}(Y ; R) \rightarrow H^{n}(X ; R)$ is trivial for any ring $R$, then the map $f_{*}: H_{n}(X ; A) \rightarrow$ $H_{n}(Y ; A)$ is trivial for any finitely generated abelian group $A$.

Proof. It is sufficient to prove the statement when $A$ is a cyclic group. First, we consider the case $A=\mathbb{Z} / p^{r}$. As in [12, p. $\left.239-240\right], \mathbb{Z} / p^{r}$ is injective in the category of $\mathbb{Z} / p^{r}$-modules. Then

$$
H^{*}\left(Z ; \mathbb{Z} / p^{r}\right) \cong \operatorname{Hom}\left(H_{*}\left(Z ; \mathbb{Z} / p^{r}\right), \mathbb{Z} / p^{r}\right)
$$

for any space $Z$. This readily implies that $f$ is trivial in homology over $\mathbb{Z} / p^{r}$.

Next, we consider the case $A=\mathbb{Z}$. By the universal coefficient theorem, for any abelian group $B$, there is a commutative diagram with exact rows:

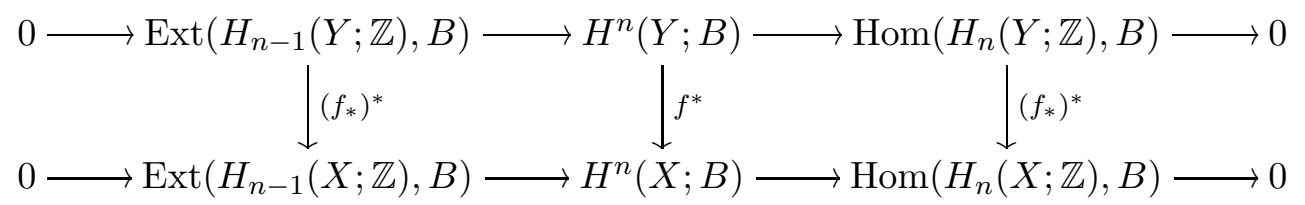

Since the middle $f^{*}$ is trivial for $B=\mathbb{Z}$ by assumption, the right $\left(f_{*}\right)^{*}$ is trivial for $B=\mathbb{Z}$. For any finitely generated abelian group $C$, there is a natural isomorphism

$$
\operatorname{Hom}(C, \mathbb{Z}) \cong \operatorname{Hom}(C / \operatorname{Tor}(C), \mathbb{Z})
$$

where $\operatorname{Tor}(C)$ denotes the torsion part of $C$. Then since $C / \operatorname{Tor}(C)$ is a free abelian group, the map $f_{*}: H_{n}(X ; \mathbb{Z}) / \operatorname{Tor}\left(H_{n}(X ; \mathbb{Z})\right) \rightarrow H_{n}(Y ; \mathbb{Z}) / \operatorname{Tor}\left(H_{n}(Y ; \mathbb{Z})\right)$ is trivial, implying $f_{*}\left(H_{n}(X ; \mathbb{Z})\right) \subset \operatorname{Tor}\left(H_{n}(Y ; \mathbb{Z})\right)$. On the other hand, the right $\left(f_{*}\right)^{*}$ is trivial for $B=$ $\mathbb{Z} / p^{r}, r$ is arbitrary. Since $H_{n}(Y ; \mathbb{Z})$ is finitely generated, if we take $r$ large enough, then we can see that the $p$-torsion part $f_{*}\left(H_{n}(X ; \mathbb{Z})\right)$ is trivial. Thus we obtain that $f_{*}: H_{n}(X ; \mathbb{Z}) \rightarrow H_{n}(Y ; \mathbb{Z})$ is trivial.

Lemma 3.3. Given a non-trivial finitely generated abelian group A, suppose that for two vertices $v, w$ of $K$, the map

$$
\left(i_{v}\right)_{*} \oplus\left(i_{w}\right)_{*}: H_{n}\left(\mathrm{dl}_{K}(v) ; A\right) \oplus H_{n}\left(\mathrm{dl}_{K}(w) ; A\right) \rightarrow H_{n}(K ; A)
$$

is not surjective. Then $\{v, w\}$ is an edge of $K$ if and only if the map

$$
\left(m_{I, J}\right)_{*}: H_{n}(K ; A) \rightarrow H_{n}\left(K_{I} * K_{J} ; A\right)
$$

is trivial for $I=\{v, w\}$ and $J=[m]-\{v, w\}$.

Proof. We set notation. The link of a vertex $u$ of $K$ is defined by

$$
\mathrm{lk}_{K}(u)=\{\sigma \in K \mid u \notin \sigma \text { and } \sigma \cup\{u\} \in K\} .
$$

For an $n$-chain $c=\sum_{i} a_{i}\left[j_{i, 0}, \ldots, j_{i, n}\right]$ of $\operatorname{lk}_{K}(u)$ for $a_{i} \in A$ and $\left[j_{i, 0}, \ldots, j_{i, n}\right] \in \mathrm{lk}_{K}(u)$, we abbreviate the $(n+1)$-chain $\sum_{i} a_{i}\left[u, j_{i, 0}, \ldots, j_{i, n}\right]$ of $K$ by $u * c$. 
Assume $\{v, w\}$ is not an edge of $K$. Let $c$ be an $n$-cycle of $K$ representing a homology class which is not in the image of the map (2). Then by the assumption above, there are $(n-1)$-chain $c_{v}$ of $\operatorname{lk}_{K}(v),(n-1)$-chain $c_{w}$ of $\operatorname{lk}_{K}(w)$ and an $n$-chain $d$ of $K_{J}$ such that

$$
c=v * c_{v}+w * c_{w}+d
$$

Since $c_{v}$ is a chain of $\mathrm{lk}_{K}(v), c_{v}$ is a chain of $K_{J}$ by the assumption above. One also gets $c_{w}$ is a chain of $K_{J}$. Since $\partial c=0$, one has $c_{v}-v * \partial c_{v}+c_{w}-w * \partial c_{w}+\partial d=0$, implying

$$
\partial c_{v}=\partial c_{w}=c_{v}+c_{w}+\partial d=0
$$

Then it follows that

$$
\left(m_{I, J}\right)_{*}([c])=\left[v * c_{v}-w * c_{v}+\partial(w * d)\right]=\left[v * c_{v}-w * c_{v}\right] \in H_{n}\left(K_{I} * K_{J} ; A\right) .
$$

Since $K_{I} * K_{J}=\Sigma K_{J}$ by assumption, the map

$$
H_{n-1}\left(K_{J} ; A\right) \rightarrow H_{n}\left(K_{I} * K_{J} ; A\right), \quad x \mapsto v * x-w * x
$$

is an isomorphism. Thus since $c_{v}$ is a cycle of $K_{J}$, if $\left(m_{I, J}\right)_{*}([c])=0$, then there is an $n$-chain $e$ of $K_{J}$ such that $c_{v}=\partial e$, implying

$$
\partial\left(w * c_{w}+d+e\right)=c_{w}+\partial d+\partial e=c_{w}+\partial d+c_{v}=0, \quad \partial\left(v * c_{v}-e\right)=c_{v}-\partial e=0 .
$$

Therefore $w * c_{w}+d+e$ and $v * c_{v}-e$ are cycles of $\mathrm{dl}_{K}(v)$ and $\mathrm{dl}_{K}(w)$, respectively, such that $[c]=\left(i_{v}\right)_{*}\left(\left[w * c_{w}+d+e\right]\right)+\left(i_{w}\right)_{*}\left(\left[v * c_{v}-e\right]\right)$. This contradicts the definition of $c$, so the only if part is proved. The if part is obvious because $H_{n}\left(K_{I} * K_{J} ; A\right)=0$.

Proposition 3.4. If $\operatorname{dim} K \leq 2$ and $K$ is Golod, then the following statements hold:

(1) the 1-skeleton of $K$ is chordal;

(2) every full subcomplex of $K$ having vertex-breakable second homology is 1-neighborly.

Proof. The first statement is proved in the proof of [9, Proposition 8.17]. Suppose that $K_{I}$ has vertex-breakable second homology. Clearly, the second statement holds for $|I| \leq 2$, and so we assume $|I| \geq 3$. Take any two vertices $v, w \in I$, and let $J=I-\{v, w\}$. Assume that $\{v, w\}$ is not an edge of $K$. Since $H^{*}\left(K_{\{v, w\}} ; R\right)$ is a free $R$-module, the strong form of the Künneth formula holds as

$$
\widetilde{H}^{n}\left(K_{\{v, w\}} * K_{J} ; R\right) \cong \bigoplus_{i+j=n-1} \widetilde{H}^{i}\left(K_{\{v, w\}} ; R\right) \otimes \widetilde{H}^{j}\left(K_{J} ; R\right) .
$$

Then it follows from Lemma 3.2 that the map $\left(m_{\{v, w\}, J}\right)_{*}: H_{2}\left(K_{I} ; A\right) \rightarrow H_{2}\left(K_{\{v, w\}} *\right.$ $\left.K_{J} ; A\right)$ is trivial for any finitely generated abelian group $A$. Thus by Lemma $3.3,\{v, w\}$ is an edge of $K_{I}$. This is a contradiction, and so $\{v, w\}$ must be an edge of $K$. 


\section{Proof of Theorem 1.7}

We will use the following simple lemmas.

Lemma 4.1. In a commutative diagram of abelian groups

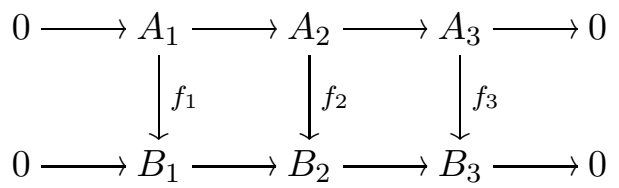

where rows are exact, suppose $f_{3}$ is injective. Then $f_{1}$ is injective if and only if so is $f_{2}$.

Proof. By the snake lemma, there is an exact sequence

$$
0 \rightarrow \operatorname{Ker} f_{1} \rightarrow \operatorname{Ker} f_{2} \rightarrow \rightarrow \operatorname{Ker} f_{3} .
$$

Since $f_{3}$ is injective, $f_{1}$ is injective if and only if so is $f_{2}$, completing the proof.

Lemma 4.2. For an exact sequence $0 \rightarrow A_{1} \rightarrow A_{2} \rightarrow A_{3} \rightarrow 0$ of abelian groups, the following statements are equivalent:

(1) the map $\operatorname{Ext}\left(A_{3}, A\right) \rightarrow \operatorname{Ext}\left(A_{2}, A\right)$ is injective for any abelian group $A$;

(2) the exact sequence $0 \rightarrow A_{1} \rightarrow A_{2} \rightarrow A_{3} \rightarrow 0$ splits.

Proof. Consider the exact sequence

$$
\begin{aligned}
0 \rightarrow \operatorname{Hom}\left(A_{3}, A\right) \rightarrow \operatorname{Hom}\left(A_{2}, A\right) \rightarrow & \operatorname{Hom}\left(A_{1}, A\right) \\
& \rightarrow \operatorname{Ext}\left(A_{3}, A\right) \rightarrow \operatorname{Ext}\left(A_{2}, A\right) \rightarrow \operatorname{Ext}\left(A_{1}, A\right) \rightarrow 0 .
\end{aligned}
$$

Then the first statement is equivalent to that the map $\operatorname{Hom}\left(A_{2}, A\right) \rightarrow \operatorname{Hom}\left(A_{1}, A\right)$ is surjective for any abelian group $A$. By setting $A=A_{1}$, this turns out to be equivalent to the second statement.

Lemma 4.3. Let $f: A \rightarrow B$ be a surjection between finitely generated abelian groups such that $f_{*}: \operatorname{Tor}\left(A, \mathbb{Z} / p^{r}\right) \rightarrow \operatorname{Tor}\left(B, \mathbb{Z} / p^{r}\right)$ is surjective for any prime $p$ and any positive integer $r$. Then $f$ admits a section.

Proof. Since $B$ is finitely generated, there is a decomposition

$$
B \cong \operatorname{Free}(B) \oplus \bigoplus_{p} \operatorname{Tor}_{p}(B)
$$

where $\operatorname{Free}(B)$ is a free abelian $\operatorname{group} \operatorname{Tor}_{p}(B)$ is the $p$-torsion part of $B$ and $p$ ranges over all primes. Since $f$ is surjective, there is a map $s$ : Free $(B) \rightarrow A$ such that $f \circ s=1_{\operatorname{Free}(B)}$. Let $g_{i}$ be a generator of $\mathbb{Z} / p^{r_{i}}$ in $\operatorname{Tor}_{p}(B) \cong \mathbb{Z} / p^{r_{1}} \oplus \cdots \oplus \mathbb{Z} / p^{r_{n}}$. Then since

$$
\operatorname{Tor}\left(B, \mathbb{Z} / p^{r}\right)=\left\{x \in B \mid p^{r} x=0\right\}
$$

and $f_{*}: \operatorname{Tor}\left(A, \mathbb{Z} / p^{r_{i}}\right) \rightarrow \operatorname{Tor}\left(B, \mathbb{Z} / p^{r_{i}}\right)$ is surjective, there is an element $h_{i}$ of $A$ such that $h_{i}$ is of order $r_{i}$ and $f\left(h_{i}\right)=g_{i}$. Thus there is a map $s_{p}$ : $\operatorname{Tor}_{p}(B) \rightarrow A$ such that $f \circ s_{p}=1_{\operatorname{Tor}_{p}(B)}$. Therefore we obtain a section $s \oplus \bigoplus_{p} s_{p}: B \rightarrow A$ of $f$. 
Lemma 4.4. Let $f: X \rightarrow Y$ be a map between finite complexes. If $f_{*}: H_{*}(X ; A) \rightarrow$ $H_{*}(Y ; A)$ is surjective for any finitely generated abelian group $A$ and $*=n-1, n$. Then $f^{*}: H^{n}(Y ; A) \rightarrow H^{n}(X ; A)$ is injective for any finitely generated abelian group $A$.

Proof. By the universal coefficient theorem, there is a commutative diagram

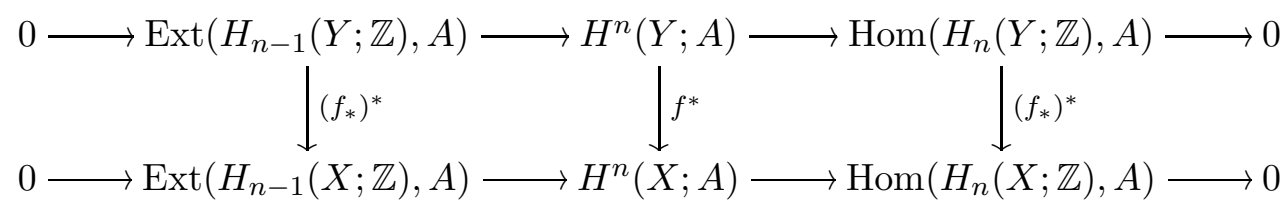

where rows are exact. By Lemma 4.1, it is sufficient to show that both left and right $\left(f_{*}\right)^{*}$ are injective. Since $f_{*}: H_{n}(X ; \mathbb{Z}) \rightarrow H_{n}(Y ; \mathbb{Z})$ is surjective, the right $\left(f_{*}\right)^{*}$ is injective. By Lemma 4.2 , the left $\left(f_{*}\right)^{*}$ is injective if and only if the map $f_{*}: H_{n-1}(X ; \mathbb{Z}) \rightarrow$ $H_{n-1}(Y ; \mathbb{Z})$ has a section. By the universal coefficient theorem, there is also a commutative diagram

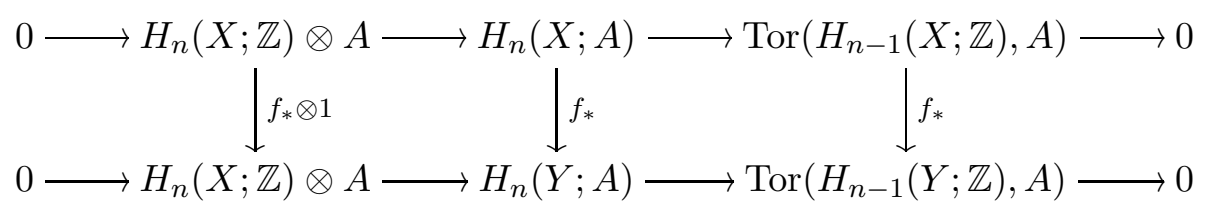

where rows are exact. Since the middle $f_{*}$ is surjective, so is the right $f_{*}$. Note that (3) holds for any abelian group $B$. Then since $H_{n-1}(X ; \mathbb{Z})$ and $H_{n-1}(Y ; \mathbb{Z})$ are finitely generated abelian groups, it follows from Lemma 4.3 that the surjectivity of the right $f_{*}$ implies the existence of a section of the map $f_{*}: H_{n-1}(X ; \mathbb{Z}) \rightarrow H_{n-1}(Y ; \mathbb{Z})$.

We apply Lemma 4.4 to our case.

Proposition 4.5. If the 1-skeleton of $K$ is chordal and the map

$$
\bigoplus_{v \in[m]}\left(i_{v}\right)_{*}: \bigoplus_{v \in[m]} H_{2}\left(\mathrm{dl}_{K}(v) ; A\right) \rightarrow H_{2}(K ; A)
$$

is surjective for any finitely generated abelian group $A$, then the map

$$
\bigoplus_{v \in[m]} i_{v}^{*}: H^{2}(K ; A) \rightarrow \bigoplus_{v \in[m]} H^{2}\left(\mathrm{dl}_{K}(v) ; A\right)
$$

is injective.

Proof. If $K$ is the boundary of a 2-simplex, then $H_{2}(K ; A)=0$ and $H^{2}(K ; A)=0$ for any abelian group $A$. Thus the statement holds obviously. Assume that $K$ is not the boundary of a 2-simplex. By Lemma 4.4, it is sufficient to show that the map

$$
\bigoplus_{v \in[m]}\left(i_{v}\right)_{*}: \bigoplus_{v \in[m]} H_{1}\left(\mathrm{dl}_{K}(v) ; A\right) \rightarrow H_{1}(K ; A)
$$

is surjective for any finitely generated abelian group $A$. For $m \leq 3, H_{1}(K ; A)=0$ since $K$ is not the boundary of a 2 -simplex, where $m$ is the number of vertices of $K$. Then 
the claim holds. For $m \geq 4$, every minimal cycle of $K$ is in $\operatorname{dl}_{K}(v)$ for some $v$ since the 1-skeleton of $K$ is chordal. Then the claim also holds.

We consider triviality of the fat-wedge filtration of $\mathbb{R} Z_{K}$.

Proposition 4.6. Suppose $\operatorname{dim} K \leq 2$ and $\varphi_{K_{I}}$ is null-homotopic for all $I \subset[m]$ with $I \neq \emptyset,[m]$. If the 1-skeleton of $K$ is chordal and the map (4) is surjective for any finitely generated abelian group $A$, then $\varphi_{K}$ is null homotopic.

Proof. By definition, $\mathbb{R} Z_{K}^{m-1}$ is path-connected, and then it has the universal cover $U_{K}$. Since $K$ is chordal, $|\widehat{K}|$ is simply-connected as in [9, Proposition 8.17]. Then by Lemma $2.4,\left(\varphi_{K}\right)_{*}: \pi_{1}(|K|) \rightarrow \pi_{1}\left(\mathbb{R} Z_{K}^{m-1}\right)$ is trivial for any basepoint of $|K|$. In particular, we get a lift $\widetilde{\varphi}_{K}:|K| \rightarrow U_{K}$. Choosing any vertex $v$ of $K$, let $L=\operatorname{dl}_{K}(v) \sqcup v$. Then by arguing verbatim as above, we get a lift $\widetilde{\varphi}_{L}:|L| \rightarrow U_{L}$ of $\varphi_{L}:|L| \rightarrow \mathbb{R} Z_{L}^{m-1}$. Since there is a commutative diagram

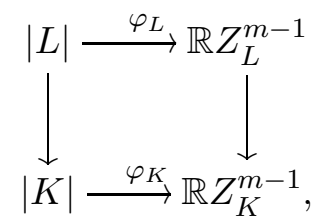

it follows from the uniqueness of lifts of $\varphi_{L}$ and $\varphi_{K}$ that the square diagram

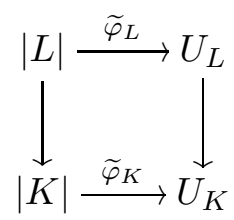

commutes. Let $A=\pi_{2}\left(\mathbb{R} Z_{K}^{m-1}\right)$ and $B=\pi_{2}\left(\mathbb{R} Z_{L}^{m-1}\right)$. Then we get a homotopy commutative diagram

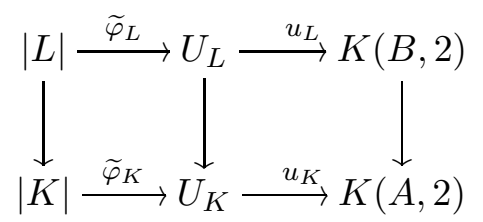

where $u_{K}$ and $u_{L}$ are isomorphisms in $\pi_{2}$. Note that $\varphi_{L}=\varphi_{\mathrm{dl}_{K}(v)} \sqcup *$. By assumption, $\varphi_{\mathrm{dl}_{K}(v)} \simeq *$. Then since $\mathbb{R} Z_{L}^{m-1}$ is path-connected, $\varphi_{L}$ is null-homotopic, implying $\widetilde{\varphi}_{L}$ is null-homotopic too. Thus the cohomology class $u_{K} \circ \widetilde{\varphi}_{K}$ belongs to the kernel of the map $H^{2}(K ; A) \rightarrow H^{2}(L ; A)$. Since this map is identified with $i_{v}^{*}: H^{2}(K ; A) \rightarrow H^{2}\left(\mathrm{dl}_{K}(v) ; A\right)$, the cohomology class $u_{K} \circ \widetilde{\varphi}_{K}$ belongs to the kernel of the map (5).

By Theorem 2.1, $\mathbb{R} Z_{K}^{m-1}$ is a suspension. Then $A \cong H_{2}\left(\mathbb{R} Z_{K} ; \mathbb{Z}\right) \otimes \mathbb{Z} \pi_{1}\left(\mathbb{R} Z_{K}^{m-1}\right)$, and in particular, $A$ is a sum of copies of $H_{2}\left(\mathbb{R} Z_{K} ; \mathbb{Z}\right)$ which is a finitely generated abelian group. Thus by Proposition 4.5, the map $i_{v}^{*}: H^{2}(K ; A) \rightarrow \bigoplus_{v \in[m]} H^{2}\left(\mathrm{dl}_{K}(v) ; A\right)$ is injective, implying $u_{K} \circ \widetilde{\varphi}_{K}$ is null-homotopic. Since $\operatorname{dim} K \leq 2$, we obtain $\varphi_{K}$ is nullhomotopic. 
Now we prove Theorem 1.7.

Proof of Theorem 1.7. The implication (1) $\Rightarrow(2)$ is proved by Proposition 3.4, and the implication $(2) \Rightarrow(3)$ is proved by Theorem 2.5 and Proposition 4.6. The implication $(3) \Rightarrow(1)$ is proved by Theorems 1.2 and 2.3 .

\section{GOLODNESS OVER FIELDS AND RINGS}

As mentioned in Section 1, it is natural to ask whether or not there is a difference between Golodness over fields and over rings. This section gives an answer by giving a simplicial complex which is Golod over any field but is not Golod over some ring.

First, we prove that the converse of Proposition 3.4 holds over a field.

Proposition 5.1. Let $\mathbb{k}$ be a field. A two-dimensional simplicial complex $K$ is Golod over $\mathbb{k}$ if and only if the following conditions hold:

(1) the 1-skeleton of $K$ is chordal;

(2) every full subcomplex of $K$ having vertex-breakable second homology over $\mathbb{k}$ is 1-neighborly.

Proof. The proof of Proposition 3.4 implies that if $K$ is Golod over $\mathbb{k}$, then the two conditions hold.

Suppose conversely that the two conditions hold. Since $\mathbb{k}$ is a field, the Künneth formula in the strong form holds as $H^{*}(X \times Y ; \mathbb{k}) \cong H^{*}(X ; \mathbb{k}) \otimes H^{*}(Y ; \mathbb{k})$. It is proved by Kätthan [11, Theorem 6.3] that for a simplicial complex $L$ of dimension at most three, the condition for (higher) Massey products in Definition 1.1 is redundant. Then to see that $K$ is Golod over $\mathbb{k}$, it is sufficient to show that the map $H^{*}\left(K_{I_{1}} * K_{I_{2}} ; \mathbb{k}\right) \rightarrow H^{*}\left(K_{I} ; \mathbb{k}\right)$ is trivial for $*=1,2$, where $I_{1}, I_{2}$ are non-empty subsets of $[m]$ such that $I_{1} \cap I_{2}=\emptyset$ and $I_{1} \cup I_{2}=I$.

Let $K^{1}$ denote the 1-skeleton of $K$. It is proved in [9, Proposition 3.2] that a graph is Golod if and only if it is chordal. Then, in particular, $K_{I}^{1}$ is Golod (over $\mathbb{k}$ ) for each $\emptyset \neq I \subset[m]$. Consider a commutative diagram

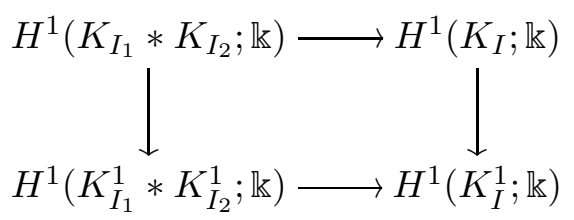

where $I_{1}, I_{2}$ are non-empty subsets of $[m]$ such that $I_{1} \cap I_{2}=\emptyset$ and $I_{1} \cup I_{2}=I$. Since $K_{I}^{1}$ is Golod over $\mathbb{k}$, the lower horizontal arrow is trivial. Then since the vertical arrows are injective, the upper horizontal arrow is trivial too.

We show that the map $H^{2}\left(K_{I_{1}} * K_{I_{2}} ; \mathbb{k}\right) \rightarrow H^{2}\left(K_{I} ; \mathbb{k}\right)$ is trivial by induction on $|I|$. For $|I|=2$, the map is trivial because $H^{2}\left(K_{I} ; \mathbb{k}\right)=0$. Suppose that $K_{J}$ is Golod for $|J|<|I|$. If $K_{I}$ has vertex-breakable second homology over $\mathbb{k}$, then it is 1-neighborly 
by assumption. Thus by Theorems 1.2, 2.3 and 2.5, $K_{I}$ is Golod over $\mathbb{k}$. Consider a commutative diagram

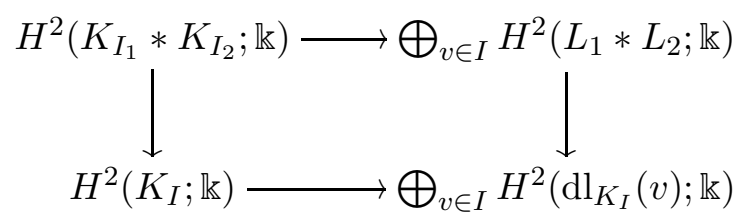

where $L_{i}=K_{I_{i}}$ for $v \notin I_{i}$ and $L_{i}=\mathrm{dl}_{K_{I_{i}}}(v)$ for $v \in I_{i}$. If $K_{I}$ does not have vertexbreakable second homology over $\mathbb{k}$, then the lower horizontal arrow is injective. By assumption, the right horizontal arrow is trivial, implying the left horizontal arrow is trivial too. Therefore the proof is complete.

Remark 5.2. The proof of Proposition 5.1 does not work over a ring in general because the Künneth formula in the strong form does not hold and $\operatorname{Hom}(-, R)$ is not right exact.

We consider the following simplicial complexes $K_{1}$ and $K_{2}$, where vertices and edges having the same labels are identified.

$K_{1}$

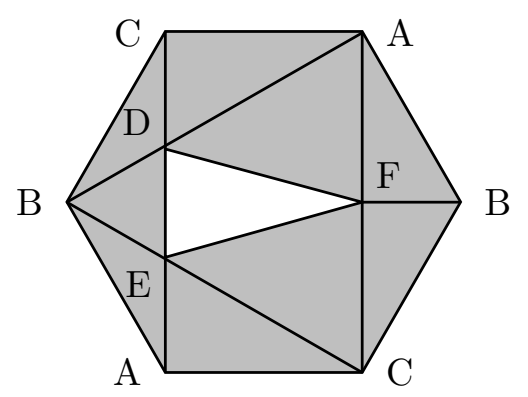

$K_{2}$

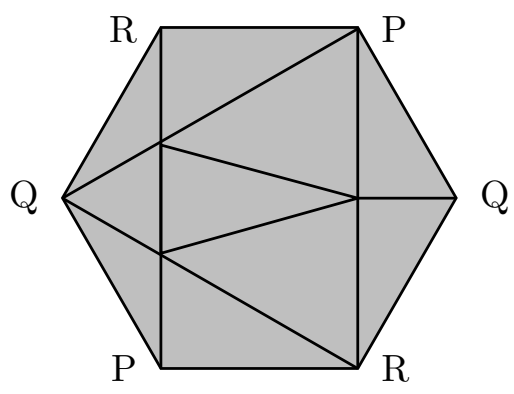

Note that

$$
\left|K_{1}\right| \simeq S^{1} \text { and }\left|K_{2}\right| \simeq \mathbb{R} P^{2} .
$$

We define a simplicial complex $M$ by gluing $K_{1}$ and $K_{2}$ along the triangles $D E F$ and $P Q R$. Since the inclusion of the triangle $D E F$ into $K_{1}$ is equivalent to the degree two self-map of $S^{1}, M$ is a triangulation of the Moore space $S^{1} \cup_{4} e^{2}$.

Proof of Theorem 1.8. Let $\mathbb{k}$ be a field of characteristic 2. By definition, $H_{2}\left(M_{I} ; \mathbb{k}\right)=0$ unless $M_{I}$ includes $K_{2}$, and $M_{I}$ does not have vertex-breakable second homology over $\mathbb{k}$ whenever $K_{2}$ is a proper subcomplex of $M_{I}$. Thus $M_{I}$ is Golod over $\mathbb{k}$ for $M_{I} \neq K_{2}$. If $M_{I}=K_{2}$, then $M_{I}$ is 1-neighborly, implying $M_{I}$ is Golod over $\mathbb{k}$ by Theorems 1.2, 2.3 and 2.5 .

Let $\mathbb{k}$ be a field of characteristic $\neq 2$. Since $H_{2}\left(M_{I} ; \mathbb{k}\right)=0$ for each $\emptyset \neq I \subset[m]$, it follows from Proposition 5.1 that $M$ is Golod over $\mathbb{k}$. Thus we obtain that $M$ is Golod over any field. 
Since $H_{2}\left(\mathrm{dl}_{M}(v) ; \mathbb{Z} / 4\right)$ is either 0 or $\mathbb{Z} / 2$ for each vertex $v$ and $H_{2}(M ; \mathbb{Z} / 4) \cong \mathbb{Z} / 4, M$ has vertex-breakable second homology over $\mathbb{Z} / 4$. Then since $M$ is not 1-neighborly, $M$ is not Golod over some ring by Theorem 1.7, where one actually sees from Lemma 3.3 that $M$ is not Golod over $\mathbb{Z} / 4$. Thus the proof is complete

\section{REFERENCES}

[1] A. Bahri, M. Bendersky, and F.R. Cohen, Polyhedral products and features of their homotopy theory, in Handbook of Homotopy Theory, Chapman and Hall/CRC, 2019.

[2] I.V. Baskakov, V.M. Buchstaber, and T.E. Panov, Cellular cochain algebras and torus actions, Russian Math. Surveys 59 (2004), no. 3, 562-563.

[3] E.S. Golod, On the homologies of certain local rings, Soviet Math. Dokl. 3 (1962), 745-748.

[4] J. Grbić and S. Theriault, The homotopy type of the complement of a coordinate subspace arrangement, Topology 46 (2007), 357-396.

[5] V. Grujić and V. Welker, Discrete Morse theory for moment-angle complexes of pairs $\left(D^{n}, S^{n-1}\right)$, Monatsh. Math. 176 (2015), no. 2, 255-273.

[6] K. Iriye and D. Kishimoto, Decompositions of polyhedral products for shifted complexes, Advances in Math. 245 (2013), 716-736.

[7] K. Iriye and D. Kishimoto, Golodness and polyhedral products for two-dimensional simplicial complexes, Forum Math. 30 (2018), no. 2, 527-532.

[8] K. Iriye and D. Kishimoto, Golodness and polyhedral products of simplicial complexes with minimal Taylor resolutions, Homology Homotopy Appl. 20 (2018), no. 1, 69-78.

[9] K. Iriye and D. Kishimoto, Fat-wedge filtration and decomposition of polyhedral products, Kyoto J. Math. 59 (2019), no. 1, 1-51.

[10] K. Iriye and T. Yano, A Golod complex with non-suspension moment-angle complex, Topology Appl. 225 (2017), no. 7, 145-163.

[11] L. Katthän, A non-Golod ring with a trivial product on its Koszul homology, J. Algebra 479 (2017), 244-262.

[12] J. Neisendorfer, Algebraic methods in unstable homotopy theory, New Mathematical Monographs 12, Cambridge University Press, Cambridge, 2010.

Department of Mathematical Sciences, Osaka Prefecture University, Sakai, 599-8531, JAPAN

Email address: kiriye@mi.s.osakafu-u.ac.jp

Department of Mathematics, Kyoto University, Kyoto, 606-8502, Japan

Email address: kishi@math.kyoto-u.ac.jp 